\title{
A CLASSIFICATION OF TORSORS OVER LAURENT POLYNOMIAL RINGS
}

\author{
V. CHERNOUSOV, P. GILLE, AND A. PIANZOLA
}

\begin{abstract}
Let $R_{n}$ be the ring of Laurent polynomials in $n$ variables over a field $k$ of characteristic zero and let $K_{n}$ be its fraction field. Given a linear algebraic $k$-group $G$, we show that a $K_{n}$-torsor under $G$ which is unramified with respect to $X=\operatorname{Spec}\left(R_{n}\right)$ extends to a unique toral $R_{n}-$ torsor under $G$. This result, in turn, allows us to classify all $G$-torsors over $R_{n}$.

Keywords: Reductive group scheme, torsor, multiloop algebra.
\end{abstract}

MSC 2000: 14F20, 20G15, 17B67, 11E72.

\section{Contents}

1. Introduction

2. Unramified functors

3. Non-abelian cohomology

3.1. Some terminology

3.2. Torsion bijection

3.3. Acyclicity Theorem

3.5. Grothendieck-Serre's conjecture

3.10. Rational torsors everywhere locally defined

4. Proof of Theorem 1.2

5. Applications

5.1. A disjoint union decomposition for $R_{n}$-torsors 11

5.6. The case of orthogonal groups 13

5.9. The projective linear case 14

5.14. Applications to $R_{n}$-Lie algebras 16

References 16

Date: July 28, 2021.

V. Chernousov was partially supported by the Canada Research Chairs Program and an NSERC research grant.

P. Gille was supported by the Romanian IDEI project PCE_2012-4-364 of the Ministry of National Education CNCS-UEFISCIDI.

A. Pianzola wishes to thank NSERC and CONICET for their continuous support. 


\section{INTRODUCTION}

Torsors are the algebraic analogues of the principal homogeneous spaces that one encounters in the theory of Lie groups. While the latter are (under natural assumptions) locally trivial (in the usual topology), this is not the case for the former: a torsor $E \rightarrow X$ under a group scheme $\mathfrak{G}$ over $X$ is not necessarily trivial (i.e., isomorphic to $\mathfrak{G}$ with $\mathfrak{G}$ acting on itself by right multiplication) when restricted to any non-empty Zariski open subset $U$ of $X$. The reason for this is that the Zariski topology is too coarse. The path out of this serious obstacle was initiated by J.-P. Serre (with what is now called the finite étale topology) and then implemented in full generality (together with an accompanying descent theory) by A. Grothendieck. The idea is to have certain morphisms $U \rightarrow X$ (e.g. étale or flat and of finite presentation) replace open immersions as trivializing local data.

Torsors have played an important role in number theory (Brauer groups, Tate-Shafarevich group, Manin obstructions) and in the Langlands program (Ngo's proof of the Fundamental Lemma). Somehow surprisingly torsors have been used over the last decade to solve difficult problems in infinite dimensional Lie theory (see GP3] for an extensive list of references. See also [CGP2] and [KLP]).

The way that torsors arise in this context is the following. The infinite dimensional object $\mathcal{L}$ under consideration (for example, the centreless core of an extended affine Lie algebra [EALA] or a Lie superconformal algebra) has an invariant called the centroid (essentially the linear endomorphisms of the object that commute with their multiplication). These centroids are Laurent polynomial rings $k\left[t_{1}^{ \pm 1}, \cdots, t_{n}^{ \pm 1}\right]$ in finitely many variables over a base field $k$ of characteristic 0 . We will denote this ring by $R_{n}$, or simply by $R$ if no confusion is possible.

The object $\mathcal{L}$ is naturally an $R$-module and it inherits the algebraic structure of $\mathcal{L}$ (for example $\mathcal{L}$ is a Lie algebra over $R$ ). It is when $\mathcal{L}$ is viewed as an object over $R$ that torsors enter into the picture. One can for example classify up to $R$-isomorphism the objects under consideration using non-abelian étale cohomology. Of course at the end of the day one wants to understand the problem under consideration (say a classification) over $k$ and not $R$. Thankfully there is a beautiful theory, known as the "centroid trick", that allows this passage.

The above discussion motivates why one is interested in the classification of torsors over $R$ under a smooth reductive $R$-group scheme. We believe that the understanding of such torsors is of its own interest. This is the purpose of the present work. There is an important class of torsors under $\mathfrak{G}$ called loop torsors that appear naturally in infinite dimensional Lie theory. (Loop torsors are defined over an arbitrary base in GP3. They have already caught the attention of researchers in other areas. See for example [PZ].) It is shown in GP3] that if $E$ is a torsor over $R$ under $\mathfrak{G}$, then $E$ being a loop torsor is equivalent to $E$ being toral, i.e. that the twisted $R$-group scheme 
$E_{\mathfrak{G}}$ admits a maximal torus. This is a remarkable property of the ring $R$ : loop and toral torsors coincide.

Toral torsors under reductive group schemes were completely classified in our paper CGP2 with the use of Bruhat-Tits theory of buildings (see the Acyclicity Theorem 3.4). We will use toral torsors in what follows (but the reader is asked to keep in mind that these are precisely the torsors that arise in infinite dimensional Lie theory). Given a smooth reductive group scheme $\mathfrak{G}$ over $R_{n}$ we want to classify/describe all the isomorphism classes of $R_{n}$-torsors under $\mathfrak{G}$. Since $\mathfrak{G}$ is smooth reductive they are in natural one-to-one correspondence with elements of the pointed set $H_{e ́ t}^{1}\left(R_{n}, \mathfrak{G}\right)$. We have of course by definition a natural inclusion

$$
H_{\text {ét,toral }}^{1}\left(R_{n}, \mathfrak{G}\right) \subseteq H_{\text {ét }}^{1}\left(R_{n}, \mathfrak{G}\right)
$$

where $H_{\text {ét,toral }}^{1}\left(R_{n}, \mathfrak{G}\right)$ is the subset consisting of (isomorphism) classes of toral $\mathfrak{G}$-torsors. From now on by default our topology will be étale; in particular we will denote $H_{e ́ t}^{1}$ by $H^{1}$ and $H_{e ́ t, t o r a l}^{1}$ by $H_{\text {toral }}^{1}$. One of our main results is the following.

1.1. Theorem. Under the above notation there is a natural bijection

$$
H^{1}\left(R_{n}, \mathfrak{G}\right) \longleftrightarrow \bigsqcup_{[E] \in H_{\text {toral }}^{1}\left(R_{n}, \mathfrak{G}\right)} H_{Z a r}^{1}\left(R_{n},{ }^{E} \mathfrak{G}\right) .
$$

The content of the above equality could be put in words as follows. Given a torsor $E^{\prime}$ over $R_{n}$ under $\mathfrak{G}$ there exists a unique toral torsor $E$ such that $E^{\prime}$ is locally isomorphic (in the Zariski topology) to $E$.

The proof of this result is achieved by a careful analysis (of independent interest) of the ramification of the torsors under consideration. We denote by $K_{n}=k\left(t_{1}, \ldots, t_{n}\right)$ the fraction field of $R_{n}$ and set $F_{n}=k\left(\left(t_{1}\right)\right) \ldots\left(\left(t_{n}\right)\right)$. The precise statement of our other main result is the following.

1.2. Theorem. Let $\mathfrak{G}$ be a smooth affine $R_{n}$-group scheme. Assume that either

(i) $\mathfrak{G}$ is reductive and admits a maximal $R_{n}$-torus (equivalently $\mathfrak{G}$ is "loop reductive" GP3, cor. 6.3]);

or

(ii) there exists a linear (smooth, not necessary connected) algebraic group $\mathbf{G}$ over $k$ and a loop torsor $E$ under $\mathbf{G} \times{ }_{k} R_{n}$ such that $\mathfrak{G}={ }^{E}\left(\mathbf{G} \times{ }_{k} R_{n}\right)$. Then we have natural bijections

$$
H_{\text {toral }}^{1}\left(R_{n}, \mathfrak{G}\right) \stackrel{\sim}{\longrightarrow} H^{1}\left(K_{n}, \mathfrak{G}\right)_{R_{n}-\text { unr }} \stackrel{\sim}{\longrightarrow} H^{1}\left(F_{n}, \mathfrak{G}\right)
$$

where $H^{1}\left(K_{n}, \mathfrak{G}\right)_{R_{n}-u n r}$ stands for the subset of the Galois cohomology set $H^{1}\left(K_{n}, \mathfrak{G}\right)$ consisting of (isomorphism) classes of $\mathfrak{G}$-torsors over $K_{n}$ extending everywhere in codimension one (see 3.10 ).

We need to explain briefly the assumptions. In both cases (i) and (ii) we consider loop (=toral) group schemes because they play a central role in the 
classification of $R_{n}$-torsors (see Theorem 1.1). Also, even though reductive group schemes are the main interest in this paper, in case (ii) we include group schemes over $R_{n}$ which are not necessary "connected". Far from being a trivial generalization, this case is absolutely essential for applications to infinite dimensional Lie theory. Indeed one is forced to understand twisted forms of $R_{n}$-Lie algebras $\mathfrak{g} \otimes_{k} R_{n}$, where $\mathfrak{g}$ is a split finite dimensional simple Lie algebra over $k$. This leads to torsors under the group $\mathbf{G} \times{ }_{k} R_{n}$ where $\mathbf{G}$ is the linear algebraic $k$-group $\operatorname{Aut}(\mathfrak{g})$. Many interesting infinite dimensional Lie objects over $k$, including extended affine Lie algebras (a particular case of them are the celebrated affine Kac-Moody Lie algebras) and Lie superconformal algebras, follow under the above considerations.

Note that the special case $\mathbf{G}=\mathrm{PGL}_{d}$ (i.e. $R_{n}$-Azumaya algebras) was already quite understood by Brauer group techniques when the base field is algebraically closed [GP2, §4.4]. Note also that Theorem 1.2 refines our acyclicity theorem, i.e. the bijection $H_{\text {toral }}^{1}\left(R_{n}, \mathfrak{G}\right) \stackrel{\sim}{\longrightarrow} H^{1}\left(F_{n}, \mathfrak{G}\right)$ (CGP2, th. 14.1] in case (i) (resp. [GP3, th. 8.1] in case (ii)).

The structure of the paper is as follows. In section 2, we establish useful generalities about unramified functors. Section 3 discusses unramified nonabelian cohomology. In section 4, we prove Theorem 1.2, Section 5 is devoted to applications including a disjoint union decomposition for the set $H^{1}\left(R_{n}, \mathfrak{G}\right)$ (Theorem 1.1). Two important particular cases are considered in detail: the cases of orthogonal groups and projective linear groups. This illustrates that our main result, which may look rather abstract and remote in appearance, can lead to new very concrete classifications/descriptions of familiar objects.

\section{UNRAMIFIED FUNCTORS}

We follow essentially the setting of [CT]. Let $S$ be a scheme. If $X$ is an integral $S$-scheme we denote by $\kappa(X)$ the fraction field of $X$. Let $\mathcal{F}$ be an $S$-functor, that is a contravariant functor $X \mapsto \mathcal{F}(X)$ from the category of $S$-schemes into the category of sets. If $X$ is integral normal one defines the following two subsets of $\mathcal{F}(\kappa(X))$ :

$$
\mathcal{F}(\kappa(X))_{X-l o c}:=\bigcap_{x \in X} \operatorname{Im}\left(\mathcal{F}\left(O_{X, x}\right) \rightarrow \mathcal{F}(\kappa(X))\right)
$$

and

$$
\mathcal{F}(\kappa(X))_{X-\text { unr }}:=\bigcap_{x \in X^{(1)}} \operatorname{Im}\left(\mathcal{F}\left(O_{X, x}\right) \rightarrow \mathcal{F}(\kappa(X))\right) .
$$

The first subset $\mathcal{F}(\kappa(X))_{X-l o c}$ is called the subset of local classes with respect to $X$ and the second one $\mathcal{F}(\kappa(X))_{X-u n r}$ is called the subset of unramified classes with respect to $X$. Obviously, we have the inclusions

$$
\mathcal{F}(\kappa(X))_{X-l o c} \subseteq \mathcal{F}(\kappa(X))_{X-u n r} \subseteq \mathcal{F}(\kappa(X)) .
$$


2.1. Lemma. Let $Y$ is an integral normal scheme over $S$. Let $f: Y \rightarrow X$ be a dominant morphism of $S$-schemes. Consider the map $\mathcal{F}\left(f^{*}\right): \mathcal{F}(\kappa(X)) \rightarrow$ $\mathcal{F}(\kappa(Y))$ induced by the comorphism $f^{*}=f_{\kappa(X)}^{*}: \kappa(X) \rightarrow \kappa(Y)$. Then.

(1) $\mathcal{F}\left(f^{*}\right)\left(\mathcal{F}(\kappa(X))_{X-l o c}\right) \subseteq \mathcal{F}(\kappa(Y))_{Y-l o c}$.

(2) If $f$ is flat then $\mathcal{F}\left(f^{*}\right)\left(\mathcal{F}(\kappa(X))_{X-u n r}\right) \subseteq \mathcal{F}(\kappa(Y))_{Y-u n r}$.

Proof. (1) The comorphism $f^{*}$ allows us to view $\kappa(X)$ as a subfield $\kappa(X) \hookrightarrow$ $\kappa(Y)$ of the field $\kappa(Y)$. Let $\gamma \in \mathcal{F}(\kappa(X))_{X-l o c}$. We want to show that its image $\gamma_{\kappa(Y)}:=\mathcal{F}\left(f^{*}\right)(\gamma) \in \mathcal{F}(\kappa(Y))$ under the base change is local with respect to $Y$. Let $y \in Y$ and put $x=f(y)$. The commutative square

$$
\begin{array}{ccc}
\kappa(X) & \subset & \kappa(Y) \\
\cup & \cup \\
O_{X, x} & \subset & O_{Y, y}
\end{array}
$$

induces a commutative diagram

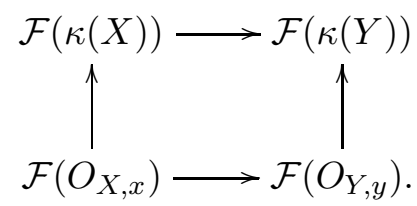

Since $\gamma \in \operatorname{Im}\left(\mathcal{F}\left(O_{X, x}\right) \rightarrow \mathcal{F}(\kappa(X))\right)$, it follows that $\gamma_{\kappa(Y)}$ is contained in $\operatorname{Im}\left(\mathcal{F}\left(O_{Y, y}\right) \rightarrow \mathcal{F}(\kappa(Y))\right)$. Thus $\gamma_{\kappa(Y)} \in \mathcal{F}(\kappa(Y))_{Y-l o c}$.

(2) Assume now that $\gamma \in \mathcal{F}(\kappa(X))_{X-u n r}$ and let $y \in Y^{(1)}$. As above, we set $x=f(y)$. Without loss of generality we may assume that

$$
X=\operatorname{Spec}(A)=\operatorname{Spec}\left(O_{X, x}\right)
$$

and that

$$
Y=\operatorname{Spec}(B)=\operatorname{Spec}\left(O_{Y, y}\right)
$$

where $B$ is a DVR. Let $v$ be the discrete valuation on $\kappa(Y)$ corresponding to the valuation $\operatorname{ring} B$. For brevity we denote by $K$ (resp. $L$ ) the fraction field of $A$ (resp. $B$ ). If $v\left(K^{\times}\right)=0$, then $K \subset B$ and therefore

$$
\gamma_{\kappa(Y)} \in \operatorname{Im}\left(\mathcal{F}\left(O_{Y, y}\right) \rightarrow \mathcal{F}(\kappa(Y))\right) .
$$

Assume now that $v\left(K^{\times}\right) \neq 0$. Then $\mathfrak{m}_{A} B \neq B$ so that $A \rightarrow B$ is a local morphism. By [EGAI, 0.6.6.2], since $B$ is flat over $A$ the $\operatorname{ring} B$ is a faithfully flat $A$-module. It follows that $A=B \cap K$ (apply [Bbk, $\S \mathrm{I} .3, \S 5$, prop. 10] with $F=K$ and $F^{\prime}=A$. An alternative proof can be given by appealing to [EGAIV, 2.1.13]). Let $A_{v}=\left\{x \in K^{\times} \mid v(x) \geq 0\right\}$ be the valuation ring of $v_{\mid K}$. Then $A_{v}=K \cap B=A$, so that $A$ is a DVR. This implies that $\gamma \in \operatorname{Im}\left(\mathcal{F}\left(O_{X, x}\right) \rightarrow \mathcal{F}(\kappa(X))\right)$, and the commutative diagram above yields that $\gamma_{\kappa(Y)} \in \operatorname{Im}\left(\mathcal{F}\left(O_{Y, y}\right) \rightarrow \mathcal{F}(\kappa(Y))\right)$. Thus $\gamma_{\kappa(Y)} \in \mathcal{F}(\kappa(Y))_{Y-u n r}$. 
We shall discuss next the case of non-abelian cohomology functors, but we remark that this technique and considerations can be applied to various interesting functors such as Brauer groups, Witt groups, unramified Galois cohomology...

\section{NON-ABELIAN COHOMOLOGY}

3.1. Some terminology. Let $X$ be a scheme and let $\mathfrak{G}$ be an $X$-group scheme. The pointed set of non-abelian Čech cohomology on the flat (resp. étale, Zariski) site of $X$ with coefficients in $\mathfrak{G}$, is denoted by $H_{\text {fppf }}^{1}(X, \mathfrak{G})$ (resp. $\left.H_{e t t}^{1}(X, \mathfrak{G}), H_{Z a r}^{1}(X, \mathfrak{G})\right)$. These pointed sets measure the isomorphism classes of sheaf torsors over $X$ under $\mathfrak{G}$ with respect to the chosen topology (see [M], Ch. IV §1] and [DG] for basic definitions and references). If $X=\operatorname{Spec}(R)$, following customary usage and depending on the context, we also use the notation $H_{f p p f}^{1}(R, \mathfrak{G})$ instead of $H_{f p p f}^{1}(X, \mathfrak{G})$. Similarly for the étale and Zariski sites.

If $\mathfrak{G}$ is in addition affine, by faithfully flat descent all of our sheaf torsors are representable. They are thus torsors in the usual sense. For a $\mathfrak{G}$-torsor $E$ we denote by ${ }^{E} \mathfrak{G}$ the twisted form of $\mathfrak{G}$ by inner automorphisms; it is an affine group scheme over $X$. Furthermore, if $\mathfrak{G}$ is smooth all torsors are locally trivial for the étale topology. In particular, $H_{\text {ét }}^{1}(X, \mathfrak{G})=H_{f p p f}^{1}(X, \mathfrak{G})$. These assumptions on $\mathfrak{G}$ hold in most of the situations that arise in our work. Also, as we mentioned in the introduction, by default our topology will be étale so that instead of $H_{e ́ t}^{1}(X, \mathfrak{G})$ we will write $H^{1}(X, \mathfrak{G})$.

Given an $X$-group $\mathfrak{G}$ and a morphisms $Y \rightarrow X$ of schemes, we let $\mathfrak{G}_{Y}$ denote the $Y$-group $\mathfrak{G} \times_{X} Y$ obtained by base change. For convenience, we will denote $H^{1}\left(Y, \mathfrak{G}_{Y}\right)$ by $H^{1}(Y, \mathfrak{G})$.

Assuming $\mathfrak{G}$ affine of finite type, a maximal torus $\mathfrak{T}$ of $\mathfrak{G}$ is a subgroup $X$-scheme $\mathfrak{T}$ of $\mathfrak{G}$ such that $\mathfrak{T} \times_{X} \overline{\kappa(x)}$ is a maximal $\overline{\kappa(x)}$-torus of $\mathfrak{G} \times_{X} \overline{\kappa(x)}$ for each point $x \in X$ [SGA3, XII.1]. Here $\overline{\kappa(x)}$ denotes an algebraic closure of $\kappa(x)$. A $\mathfrak{G}$-torsor $E$ is toral if the twisted group scheme ${ }^{E} \mathfrak{G}$ admits a maximal $X$-torus. We denote by $H_{\text {fppf,toral }}^{1}(X, \mathfrak{G})$ the subset of $H_{\text {fppf }}^{1}(X, \mathfrak{G})$ consisting of (isomorphism) classes of toral $X$-torsors under $\mathfrak{G}$.

3.2. Torsion bijection. If $E$ is an $X$-torsor under $\mathfrak{G}$ (not necessarily toral), according to [Gi, III.2.6.3.1] there exists a natural bijection

$$
\tau_{E}: H_{\text {fppf }}^{1}\left(X,{ }^{E} \mathfrak{G}\right) \rightarrow H_{f p p f}^{1}(X, \mathfrak{G}),
$$

called the torsion bijection, which takes the class of the trivial torsor under ${ }^{E} \mathfrak{G}$ to the class of $E$. It is easy to see that its restriction to classes of toral torsors induces a bijection

$$
H_{\text {fppf,toral }}^{1}\left(X,{ }^{E} \mathfrak{G}\right) \rightarrow H_{\text {fppf,toral }}^{1}(X, \mathfrak{G}) .
$$


3.3. Acyclicity Theorem. The following theorem is the main tool for proving our main results.

3.4. Theorem. Let $\mathfrak{G}$ be a smooth affine $R_{n}$-group scheme. Assume that either

(i) $\mathfrak{G}$ is reductive and admits a maximal $R_{n}$-torus (equivalently $\mathfrak{G}$ is "loop reductive" [GP3, cor. 6.3]);

or

(ii) there exists a linear (smooth, not necessary connected) algebraic group $\mathbf{G}$ over $k$ and a loop torsor $E$ under $\mathbf{G} \times{ }_{k} R_{n}$ such that $\mathfrak{G}={ }^{E}\left(\mathbf{G} \times{ }_{k} R_{n}\right)$.

Then a natural map

$$
H_{\text {toral }}^{1}\left(R_{n}, \mathfrak{G}\right) \longrightarrow H^{1}\left(F_{n}, \mathfrak{G}\right)
$$

is bijective.

Proof. See [CGP2, th. 14.1] in case (i) and [GP3, th. 8.1] in case (ii).

3.5. Grothendieck-Serre's conjecture. The following conjecture is due to Grothendieck-Serre ([Se, Remarque 3], [Gr, Remarque 1.1.a]).

3.6. Conjecture. Let $R$ be a regular local ring with fraction field $K$. If $\mathfrak{G}$ a reductive group scheme over $R$ then the natural map $H^{1}(R, \mathfrak{G}) \rightarrow H^{1}(K, \mathfrak{G})$ has trivial kernel.

If $R$ contains an infinite field $k$ (of any characteristic), the conjecture has been proven by Fedorov-Panin [FP, PSV]. When $\mathfrak{G}=\mathbf{G} \times_{k} R$ for some reductive $k$-group $\mathbf{G}$, the so called "constant" case, was established before by Colliot-Thélène and Ojanguren [CTO]. For our considerations we need a similar result for group schemes which are not necessary "connected".

3.7. Lemma. Let $R$ be a regular local ring with fraction field $K$. Let $\mathfrak{G}$ be an affine smooth group scheme over $R$ which is an extension of a finite twisted constant group scheme $\mathbf{F}$ over $R$ by a reductive group scheme $\mathfrak{G}^{0}$ over $R$. Assume that Grothendieck-Serre's conjecture holds for $\mathfrak{G}^{0}$. Then the map $H^{1}(R, \mathfrak{G}) \rightarrow H^{1}(K, \mathfrak{G})$ has trivial kernel.

Proof. We consider the commutative exact diagram of pointed sets

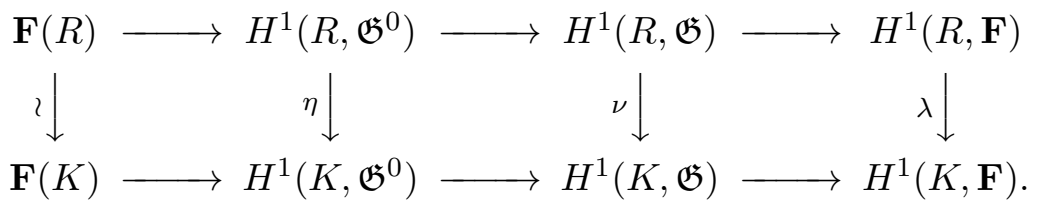

Since $\lambda$ is injective, an easy diagram chase shows that Ker $\eta$ surjects onto Ker $\nu$. But by hypothesis Ker $\eta$ vanishes, so the assertion follows.

As a corollary of Fedorov-Panin's theorem we get the following facts. 
3.8. Corollary. Let $R$ be a regular local ring containing an infinite field with fraction field $K$. Let $\mathfrak{G}$ be an affine smooth group scheme over $R$ which is an extension of a finite twisted constant group scheme over $R$ by a reductive group scheme over $R$. Then the natural map $H^{1}(R, \mathfrak{G}) \rightarrow H^{1}(K, \mathfrak{G})$ has trivial kernel.

3.9. Corollary. Let $X$ be an integral smooth affine variety over an infinite field with function field $K$. Let $\mathfrak{G}$ be an affine smooth group scheme over $X$ which is an extension of a finite twisted constant group scheme over $X$ by a reductive group scheme over $X$. Then the sequence of pointed sets

$$
1 \longrightarrow H_{Z a r}^{1}(X, \mathfrak{G}) \longrightarrow H^{1}(X, \mathfrak{G}) \longrightarrow H^{1}(K, \mathfrak{G})
$$

is exact.

3.10. Rational torsors everywhere locally defined. For a smooth affine group scheme $\mathfrak{G}$ over an integral normal scheme $X$ Colliot-Thélène and Sansuc [CTS, §6] introduced the following sets:

$$
\begin{gathered}
D^{\mathfrak{G}}(X):=\operatorname{Im}\left(H^{1}(X, \mathfrak{G}) \rightarrow H^{1}(\kappa(X), \mathfrak{G})\right), \\
H^{1}(\kappa(X), \mathfrak{G})_{X-l o c}:=\bigcap_{x \in X} D^{\mathfrak{G}}\left(\mathcal{O}_{X, x}\right) \subseteq H^{1}(\kappa(X), \mathfrak{G}),
\end{gathered}
$$

and

$$
H^{1}(\kappa(X), \mathfrak{G})_{X-u n r}:=\bigcap_{x \in X^{(1)}} D^{\mathfrak{G}}\left(\mathcal{O}_{X, x}\right) \subseteq H^{1}(\kappa(X), \mathfrak{G}) .
$$

Clearly, we have the inclusions

$$
D^{\mathfrak{G}}(X) \subseteq H^{1}(\kappa(X), \mathfrak{G})_{X-l o c} \subseteq H^{1}(\kappa(X), \mathfrak{G})_{X-u n r}
$$

In our terminology introduced in $\S 2$ the two last sets are nothing but the local and unramified subsets with respect to $X$ attached to the functor $\mathcal{F}$ given by $\mathcal{F}(Y)=H^{1}(Y, \mathfrak{G})$ for each $X$-scheme $Y$. Unramified classes have the following geometrical characterization.

3.11. Lemma. Let $\gamma \in H^{1}(\kappa(X), \mathfrak{G})$. Then $\gamma \in H^{1}(\kappa(X), \mathfrak{G})_{X-\text { unr }}$ if and only if there exists an open subset $U$ of $X$ and a class $\widetilde{\gamma} \in H_{\text {ét }}^{1}(U, \mathfrak{G})$ such that

(i) $\gamma=(\widetilde{\gamma})_{\kappa(X)}$;

(ii) $X^{(1)} \subset U$.

Proof. In one direction the statement is obvious. The other one was treated in [GP2, cor. A.8].

A special case of Lemma 2.1 is then the following.

3.12. Lemma. Let $f: Y \rightarrow X$ be a dominant morphism of integral and normal $S$-schemes. Let

$$
\mathcal{F}\left(f^{*}\right): H^{1}(\kappa(X), \mathfrak{G}) \rightarrow H^{1}\left(\kappa(Y), \mathfrak{G}_{Y}\right)
$$

be the map induced by the comorphism $f^{*}: \kappa(X) \rightarrow \kappa(Y)$. Then. 
(1) $\mathcal{F}\left(f^{*}\right)\left(H^{1}(\kappa(X), \mathfrak{G})_{X-l o c}\right) \subseteq H^{1}(\kappa(Y), \mathfrak{G})_{Y-l o c}$.

(2) If $f$ is flat then

$$
\mathcal{F}\left(f^{*}\right)\left(H^{1}(\kappa(X), \mathfrak{G})_{X-u n r}\right) \subseteq H^{1}(\kappa(Y), \mathfrak{G})_{Y-u n r} .
$$

3.13. Remark. If $X$ is regular and the "purity conjecture" holds for $\mathfrak{G}$ and local rings of $X$, i.e. $D_{\mathfrak{G}}\left(O_{X, x}\right)=H^{1}(\kappa(X), \mathfrak{G})_{O_{X, x}-\text { unr }}$ for all points $x \in X$, then assertion (1) implies that (2) holds without flatness assumption for $f$.

We now combine earlier work by Colliot-Thélène/Sansuc and Nisnevich theorem [N] on Grothendieck-Serre conjecture for reductive groups over DVR.

3.14. Proposition. Assume that $X$ is a regular integral scheme and that $\mathfrak{G}$ is an extension of a finite twisted constant group scheme by a reductive group scheme $\mathfrak{G}^{0}$. Then

(1) $D^{\mathfrak{G}}$ defines a contravariant functor for the category of regular integral $X$-schemes.

(2) If $X=\operatorname{Spec}(k)$, then $H^{1}(, \mathfrak{G})_{l o c}$ defines a contravariant functor for the category of smooth integral $k$-varieties.

Proof. Nisnevich's theorem states that if $A$ is a DVR and $\mathbf{G}$ is a reductive group over $A$ then the natural map $H^{1}(R, \mathbf{G}) \rightarrow H^{1}(K, \mathbf{G})$, where $K$ is a fraction field of $A$, is injective. By Lemma 3.7, it holds more generally for a group $\mathbf{G}$ which is an extension of a finite twisted constant group by a reductive group. In particular, if $\gamma_{1}, \gamma_{2} \in H^{1}(A, \mathbf{G})$ have the same image in $H^{1}(K, \mathbf{G})$, they have the same specialization modulo the maximal ideal of A. By CTS, 6.6.1], this specialization property holds more generally over an arbitrary valuation ring. Then the assertions follow from [CTS, 6.6.3] and [CT, proposition 2.1.10].

\section{Proof of Theorem 1.2}

Let $\mathfrak{G}$ be a smooth affine group scheme over $R_{n}$ satisfying condition (i) or (ii) in Theorem 1.2. Clearly,

$$
\operatorname{Im}\left[H^{1}\left(R_{n}, \mathfrak{G}\right) \rightarrow H^{1}\left(K_{n}, \mathfrak{G}\right)\right] \subset H^{1}\left(K_{n}, \mathfrak{G}\right)_{R_{n}-\text { unr }},
$$

so that we have the factorization

$$
H_{\text {toral }}^{1}\left(R_{n}, \mathfrak{G}\right) \stackrel{\phi}{\longrightarrow} H^{1}\left(K_{n}, \mathfrak{G}\right)_{R_{n}-\text { unr }} \stackrel{\psi}{\longrightarrow} H^{1}\left(F_{n}, \mathfrak{G}\right) .
$$

The Acyclicity Theorem 3.4 states that the composite map $\psi \circ \phi$ is bijective; in particular, $\phi$ is injective and $\psi$ is surjective.

4.1. Lemma. The following are equivalent.

(i) $\phi$ is bijective.

(ii) The map

$$
{ }^{E} \psi: H^{1}\left(K_{n},{ }^{E} \mathfrak{G}\right)_{R_{n}-u n r} \longrightarrow H^{1}\left(F_{n},{ }^{E} \mathfrak{G}\right)
$$

has trivial kernel for all toral $R_{n}$-torsors $E$ under $\mathfrak{G}$. 
Proof. $(i) \Longrightarrow(i i)$ : Assume that $\phi$ is bijective. The above factorization $(*)$ and the bijectivity of $\psi \circ \phi$ yield that we have bijections

$$
H_{\text {toral }}^{1}\left(R_{n}, \mathfrak{G}\right) \stackrel{\sim}{\longrightarrow} H^{1}\left(K_{n}, \mathfrak{G}\right)_{R_{n}-\text { unr }} \stackrel{\sim}{\longrightarrow} H^{1}\left(F_{n}, \mathfrak{H}\right) .
$$

Let now $E$ be a toral $R_{n}$-torsor under $\mathfrak{G}$. Recall that the torsion bijection map

$$
\tau_{E}: H^{1}\left(R_{n},{ }^{E} \mathfrak{G}\right) \stackrel{\sim}{\longrightarrow} H^{1}\left(R_{n}, \mathfrak{G}\right)
$$

induces a bijection

$$
H_{\text {toral }}^{1}\left(R_{n},{ }^{E} \mathfrak{G}\right) \stackrel{\sim}{\longrightarrow} H_{\text {toral }}^{1}\left(R_{n}, \mathfrak{G}\right)
$$

(see $\S 3.2$ ). The commutative diagram of torsion bijections

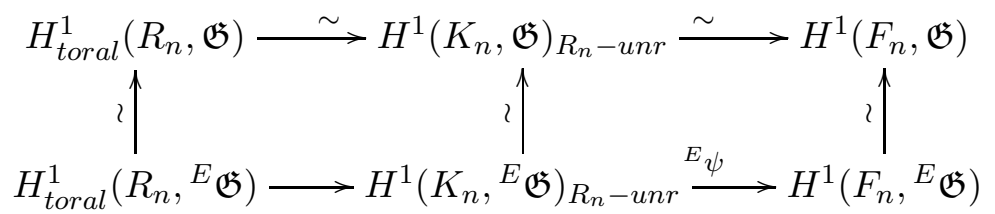

shows that ${ }^{E} \psi$ is bijective, so a fortiori has trivial kernel.

$($ ii $) \Longrightarrow(i)$ : We have noticed above that $\phi$ is injective, hence it remains to prove its surjectivity only. Let $\left[E^{\prime}\right] \in H^{1}\left(K_{n}, \mathfrak{G}\right)_{R_{n}-\text { unr. Since } \psi \circ \phi \text { is }}$ bijective there exists a class $[E] \in H^{1}\left(R_{n}, \mathfrak{G}\right)$ such that $\psi\left(\left[E^{\prime}\right]\right)=\psi(\phi([E]))$. It follows from the above commutative diagram that under the torsion bijection $H^{1}\left(K_{n},{ }^{E} \mathfrak{G}\right)_{R_{n}-\text { unr }} \rightarrow H^{1}(K, \mathfrak{G})_{R_{n}-\text { unr }}$ the class $\left[E^{\prime}\right]$ corresponds to an element in $H^{1}\left(K_{n},{ }^{E} \mathfrak{G}\right)_{R_{n}-u n r}$ lying in the kernel of ${ }^{E} \psi$. Since by our hypothesis $\operatorname{Ker}\left({ }^{E} \psi\right)=1$, this implies that the class $\left[E^{\prime}\right]$ corresponds to the trivial one in $H^{1}\left(K_{n},{ }^{E} \mathfrak{G}\right)_{R_{n}-u n r}$ or equivalently $\phi([E])=\left[E^{\prime}\right]$.

Note that hypothesis (i) and (ii) in Theorem 1.2 are stable with respect to twisting by a toral $R_{n}$-torsor under $\mathfrak{G}$. Therefore the above lemma reduces the proof of Theorem 1.2 to showing that for all group schemes $\mathfrak{H}$ over $R_{n}$ satisfying conditions (i) or (ii) in Theorem 1.2 a natural map

$$
\psi: H^{1}\left(K_{n}, \mathfrak{H}\right)_{R_{n}-u n r} \longrightarrow H^{1}\left(F_{n}, \mathfrak{H}\right)
$$

has trivial kernel. To prove this fact we proceed by induction on $n \geq 1$ by allowing the base field $k$ to vary.

$n=1$ : Since we are in dimension one, by Lemma 3.11 the map

$$
H^{1}\left(R_{1}, \mathfrak{H}\right) \longrightarrow H^{1}\left(K_{1}, \mathfrak{H}\right)_{R_{1}-\text { unr }}
$$

is onto. Therefore, Lemma 4.1 applied to the group scheme $\mathfrak{G}=\mathfrak{H}$ and the trivial torsor $E=1$ shows that $\psi$ has trivial kernel.

$n \geq 2$ : Consider the following field tower:

$$
K_{n} \subset F_{n-1}\left(t_{n}\right) \subset F_{n} .
$$


Let $\gamma \in \operatorname{Ker}(\psi)$ and let $\gamma^{\prime}$ be its image in $H^{1}\left(F_{n-1}\left(t_{n}\right), \mathfrak{H}\right)$. Since the morphism of affine schemes $\operatorname{Spec}\left(F_{n-1}\left[t_{n}^{ \pm 1}\right]\right) \rightarrow \operatorname{Spec}\left(R_{n}\right)$ is flat and dominant, by Lemma $3.12(2)$ we have

$$
\gamma^{\prime} \in H^{1}\left(F_{n-1}\left(t_{n}\right), \mathfrak{H}\right)_{F_{n-1}\left[t_{n}^{ \pm 1}\right]-u n r} .
$$

Since $F_{n}=F_{n-1}\left(\left(t_{n}\right)\right)$ and $\gamma_{F_{n}}=1$, we then conclude that

$$
\gamma^{\prime} \in \operatorname{Ker}\left(H^{1}\left(F_{n-1}\left(t_{n}\right), \mathfrak{H}\right)_{F_{n-1}\left[t_{n}^{ \pm 1}\right]-u n r} \rightarrow H^{1}\left(F_{n-1}\left(\left(t_{n}\right)\right), \mathfrak{H}\right)\right) .
$$

But according to the case $n=1$ (applied to the base field $F_{n-1}$ ) the last kernel is trivial. Thus $\gamma^{\prime}=1$, i.e.

$$
\gamma \in \operatorname{Ker}\left(H^{1}\left(K_{n}, \mathfrak{H}\right)_{R_{n}-\text { unr }} \rightarrow H^{1}\left(F_{n-1}\left(t_{n}\right), \mathfrak{H}\right)\right) .
$$

Now, we observe that the field $F_{n-1}\left(t_{n}\right)=k\left(\left(t_{1}\right)\right) \ldots\left(\left(t_{n-1}\right)\right)\left(t_{n}\right)$ embeds into $k\left(t_{n}\right)\left(\left(t_{1}\right)\right) \ldots\left(\left(t_{n-1}\right)\right)=k^{\prime}\left(\left(t_{1}\right)\right) \ldots\left(\left(t_{n-1}\right)\right)$ with $k^{\prime}=k\left(t_{n}\right)$, so that we have a commutative diagram

$$
\begin{gathered}
H^{1}\left(K_{n}, \mathfrak{H}\right)_{R_{n}-u n r} \quad \longrightarrow \quad H^{1}\left(F_{n-1}\left(t_{n}\right), \mathfrak{H}\right) \\
\cap \\
H^{1}\left(k^{\prime}\left(t_{1}, \ldots, t_{n-1}\right), \mathfrak{H}\right)_{R_{n-1} \otimes_{k} k^{\prime}-u n r} \longrightarrow H^{1}\left(k^{\prime}\left(\left(t_{1}\right)\right) \ldots\left(\left(t_{n-1}\right)\right), \mathfrak{H}\right),
\end{gathered}
$$

where the left vertical inclusion again is due to Lemma 3.12(2). By the induction hypothesis applied to the base field $k^{\prime}$, the bottom horizontal map has trivial kernel. Therefore the top horizontal map has trivial kernel as well. By $(* *)$, this implies $\gamma=1$.

\section{Applications}

5.1. A disjoint union decomposition for $R_{n}$-torsors. We shall use now our Theorem 1.2 and Fedorov-Panin's theorem to prove Theorem 1.1. In fact we will prove a little bit more general result by allowing $\mathfrak{G}$ to be any group scheme satisfying conditions of Theorem 1.2 .

5.2. Theorem. Let $\mathfrak{G}$ be as in Theorem 1.2. Then there is a natural bijection

$$
\bigsqcup_{[E] \in H_{\text {toral }}^{1}\left(R_{n}, \mathfrak{G}\right)} H_{Z \text { ar }}^{1}\left(R_{n},{ }^{E} \mathfrak{G}\right) \stackrel{\Theta}{\stackrel{\leftrightarrow}{\longrightarrow}} H^{1}\left(R_{n}, \mathfrak{G}\right) .
$$

Proof. Recall first that the torsion bijection $\tau_{E}$ (see $\S 3.2$ ) allows us to embed

$$
H_{Z a r}^{1}\left(R_{n},{ }^{E} \mathfrak{G}\right) \hookrightarrow H^{1}\left(R_{n},{ }^{E} \mathfrak{G}\right) \stackrel{\tau_{E}}{\longrightarrow} H^{1}\left(R_{n}, \mathfrak{G}\right) .
$$

and this, in turn, induces a natural map

$$
\Theta: \bigsqcup_{[E] \in H_{\text {toral }}^{1}\left(R_{n}, \mathfrak{G}\right)} H_{Z a r}^{1}\left(R_{n},{ }^{E} \mathfrak{G}\right) \rightarrow H_{e ́ t}^{1}\left(R_{n}, \mathfrak{G}\right) .
$$

Surjectivity of $\Theta$. Assume $[\gamma] \in H^{1}\left(R_{n}, \mathfrak{G}\right)$. Since the generic class $\gamma_{K_{n}} \in$ $H^{1}\left(K_{n}, \mathfrak{G}\right)$ is $R_{n}$-unramified by Theorem 1.2 there is a unique toral class 
$[E] \in H^{1}\left(R_{n}, \mathfrak{G}\right)_{\text {toral }}$ such that $[E]_{K_{n}}=\gamma_{K_{n}}$. Consider the following commutative diagram

$$
\begin{aligned}
H^{1}\left(R_{n}, \mathfrak{G}\right) & \longrightarrow H^{1}\left(K_{n}, \mathfrak{G}\right) \\
\tau_{E} \uparrow \imath & \tau_{E} \uparrow \imath \\
H_{Z a r}^{1}\left(R_{n},{ }^{E} \mathfrak{G}\right) \stackrel{\iota}{\hookrightarrow} H^{1}\left(R_{n},{ }^{E} \mathfrak{G}\right) & \longrightarrow H^{1}\left(K_{n},{ }^{E} \mathfrak{G}\right)
\end{aligned}
$$

with an exact bottom horizontal line (see Corollary [3.9). It follows from the diagram that that $\tau_{E}^{-1}(\gamma) \in \operatorname{Im} \iota$. Hence there exists a unique class $\eta \in H_{Z a r}^{1}\left(R_{n},{ }^{E} \mathfrak{G}\right)$ such that $\eta=\tau_{E}^{-1}(\gamma)$. By construction, $\Theta(\eta)=\gamma$.

Injectivity of $\Theta$. Let $E, E^{\prime}$ be two toral torsors under $\mathfrak{G}$ and let

$$
\eta \in H_{Z a r}^{1}\left(R_{n},{ }^{E} \mathfrak{G}\right), \quad \eta^{\prime} \in H_{Z a r}^{1}\left(R_{n},{ }^{E^{\prime}} \mathfrak{G}\right)
$$

be such that $\Theta(\eta)=\Theta\left(\eta^{\prime}\right)$, i.e. $\tau_{E}(\eta)=\tau_{E^{\prime}}\left(\eta^{\prime}\right) \in H^{1}\left(R_{n}, \mathfrak{G}\right)$. Since $\eta$ is locally trivial in the Zariski topology we conclude that

$$
\tau_{E}(\eta)_{K_{n}}=\tau_{E}(1)_{K_{n}}=[E]_{K_{n}}
$$

and similarly for $E^{\prime}$. It follows that

$$
[E]_{K_{n}}=\left[E^{\prime}\right]_{K_{n}} \in H^{1}\left(K_{n}, \mathfrak{G}\right)_{R_{n}-u n r}
$$

and therefore, by Theorem 1.2 , we have $[E]=\left[E^{\prime}\right]$. But $\tau_{E}=\tau_{E^{\prime}}$ is bijective. Therefore, $\eta=\eta^{\prime}$.

5.3. Example. Let $\mathbf{G}$ be a reductive $k$-group with the property that all of its semisimple quotients are isotropic (for example, $k$-split). By a result of Raghunathan $[\mathrm{R}$, th. B] one has

$$
H_{Z a r}^{1}\left(\mathbf{A}_{k}^{n}, \mathbf{G}\right)=1
$$

for all $n \geq 0$. According to GP2, prop. 2.2], any Zariski locally trivial $\mathbf{G}$-torsor over $R_{n}$ can be extended to a $\mathbf{G}$-torsor over $\mathbf{A}_{k}^{n}$. Therefore $H_{Z a r}^{1}\left(R_{n}, \mathbf{G}\right)=1$. This implies that the map $H^{1}\left(R_{n}, \mathbf{G}\right) \rightarrow H^{1}\left(K_{n}, \mathbf{G}\right)$ has trivial kernel. In other words, rationally trivial $R_{n}$-torsors under $\mathbf{G}$ are trivial.

We expect that the similar result holds in a more general case.

5.4. Conjecture. Let $\mathfrak{G}$ be a loop reductive group scheme over $R_{n}$. If all semisimple quotients of $\mathfrak{G}$ are isotropic, then $H_{Z a r}^{1}\left(R_{n}, \mathfrak{G}\right)=1$.

5.5. Remark. Note that Artamonov's freeness result [A] as well as Parimala's result $[\mathrm{P}]$ for quadratic forms over $R_{2}$ are particular special cases of this conjecture

Theorem 5.2 gives a classification of all $\mathfrak{G}$-torsors that involves first the description of all its toral torsors and then to studying locally trivial in Zariski topology torsors under its twisted toral forms. In the following two subsections we show how our theorem works in particular cases of orthogonal groups and projective linear groups. 
5.6. The case of orthogonal groups. Let $q_{\text {split }}$ be a split quadratic form over $k$ of dimension $d \geq 1$ and let $\mathbf{O}\left(q_{\text {split }}\right)$ be the corresponding split orthogonal group. It is well-known that $H^{1}\left(R_{n}, \mathbf{O}\left(q_{\text {split }}\right)\right)$ classifies non-singular quadratic $R_{n}$-forms of dimension $d$ [DG, III.5.2]. This allows us to identify classes of torsors under $\mathfrak{G}=\mathbf{O}\left(q_{\text {split, } R_{n}}\right)$ with classes of $d$-dimensional quadratic forms over the ring $R_{n}$.

5.7. Proposition. For each subset $I \subseteq\{1, \ldots, d\}$, we put $t_{I}=\prod_{i \in I} t_{i} \in R_{n}^{\times}$ with the convention $t_{\emptyset}=1$.

(1) Each class in $H_{\text {toral }}^{1}\left(R_{n}, \mathbf{O}\left(q_{\text {split }}\right)\right)$ contains a unique $R_{n}$-quadratic form of the shape

$$
\bigoplus_{I \subseteq\{1, \ldots, d\}}\left\langle t_{I}\right\rangle \otimes q_{I, R_{n}}
$$

where all $q_{I}$ 's with $I \neq \emptyset$ are non-singular anisotropic quadratic forms over $k$ such that

$$
d=\bigoplus_{I \subseteq\{1, \ldots, d\}} \operatorname{dim}\left(q_{I}\right)
$$

(2) Let $q$ be a non-singular quadratic $R_{n}$-form of dimension $d$. Then there exists a unique quadratic $R_{n}$-form $q_{\text {loop }}$ as in (1) such that $q$ is a Zariski $R_{n}$-form of $q_{\text {loop. }}$. Furthermore, $q$ is isometric to $q_{\text {loop }}$ if and only if $q$ is diagonalizable.

Proof. (1) By Acyclicity Theorem 3.4 it suffices to compute $H^{1}\left(F_{n}, \mathbf{O}\left(q_{\text {split }}\right)\right)$ or equivalently isometry classes of $d$-dimensional quadratic forms over $F_{n}$.

Let $q$ be such quadratic form. We want to show that it is as in (1). By the Witt theorem we may assume without loss of generality that $q$ is anisotropic. We proceed by induction on $n \geq 0$. The case $n=0$ is obvious. Assume that $n \geq 1$. Note that $F_{n}=F_{n-1}\left(\left(t_{n}\right)\right)$. Springer's decomposition [EKM, §19], then shows that $q \cong q^{\prime} \oplus\left\langle t_{n}\right\rangle q^{\prime \prime}$ where $q^{\prime}$ and $q^{\prime \prime}$ are (unique) anisotropic quadratic forms over $F_{n-1}$. By induction on $n, q^{\prime}$ and $q^{\prime \prime}$ are of the required form, hence we the assertion for $q$ follows. The unicity is clear.

(2) The first assertion follows from Theorem 5.2. If $q$ is isometric to $q_{\text {loop }}$, then $q$ is diagonalizable since so is $q_{l o o p}$. Conversely, assume that $q$ is diagonalizable: $q=\left\langle b_{1}, \ldots, b_{d}\right\rangle$. Since

$$
R_{n}^{\times} /\left(R_{n}^{\times}\right)^{2} \stackrel{\sim}{\longrightarrow} k^{\times} /\left(k^{\times}\right)^{2} \times\left\langle t_{1}^{\epsilon_{1}} \ldots t_{n}^{\epsilon_{n}} \mid \epsilon_{1}, \ldots, \epsilon_{n}=0,1\right\rangle .
$$

all coefficients of $q$ are of the shape $b_{i}=a_{i} t_{I_{i}}$ with $I_{i} \subseteq\{1, \ldots, d\}$ and $a_{i} \in k^{\times}$. Then we can renumber $b_{1}, \ldots, b_{d}$ in such a way that $q$ is as in (1) and we are done.

5.8. Corollary. Toral classes in $H^{1}\left(R_{n}, \mathbf{O}\left(q_{\text {split }}\right)\right)$ correspond to diagonalizable $R_{n}$-quadratic forms. 
5.9. The projective linear case. Let $\mathfrak{G}=\mathrm{PGL}_{d}$. The set $H^{1}\left(R_{n}, \mathrm{PGL}_{d}\right)$ classifies Azumaya $R_{n}$-algebras of degree $d$ [CF prop. 2.5.3.13]. Recall also that if $\mathcal{A}$ is an Azumaya algebra over $R_{n}$ of degree $d$, then there is a oneto-one correspondence between commutative étale $R_{n}$-subalgebras of $\mathcal{A}$ of dimension $d$ and maximal $R_{n}$-tori of the group scheme $\operatorname{PGL}_{1}(\mathcal{A})$. (This is a general well-known fact [SGA3, XIV.3.21.(b)]. Indeed, if $S$ is a commutative étale $R_{n}$-subalgebra of $\mathcal{A}$ of degree $d$ then $\mathfrak{T}=R_{S / R_{n}}\left(\mathbf{G}_{m}\right) / \mathbf{G}_{m}$ is a maximal $R_{n}$-torus of $\mathrm{PGL}_{1}(\mathcal{A})$. Conversely, to a maximal $R_{n}$-subtorus $\mathfrak{T}$ of $\mathrm{PGL}_{1}(\mathcal{A})$ one associates the $R_{n}$-subalgebra $\mathcal{A}^{\mathfrak{T}}$ of fixed points under the natural action of $\mathfrak{T}$. This subalgebra $\mathcal{A}^{\mathfrak{T}}$ has the required properties locally and hence globally).

From the above discussion it follows that $H_{\text {toral }}^{1}\left(R_{n}, \mathrm{PGL}_{d}\right)$ consists of isomorphism classes of Azumaya $R_{n}$-algebras $\mathcal{A}$ of degree $d$ having étale commutative $R_{n}$-subalgebras of dimension $d$.

We pass to description of locally trivial torsors under $\operatorname{PGL}_{1}(\mathcal{A})$.

5.10. Lemma. Let $\mathcal{A}$ be an Azumaya algebra over $R_{n}$. Then the natural map $H_{Z a r}^{1}\left(R_{n}, \mathrm{GL}_{1}(\mathcal{A})\right) \rightarrow H_{Z a r}^{1}\left(R_{n}, \mathrm{PGL}_{1}(\mathcal{A})\right)$ is bijective.

Proof. The exact sequence

$$
1 \rightarrow \mathrm{G}_{m} \rightarrow \mathrm{GL}_{1}(\mathcal{A}) \rightarrow \mathrm{PGL}_{1}(\mathcal{A}) \rightarrow 1
$$

gives rise to a commutative diagram with exact rows (of pointed sets)

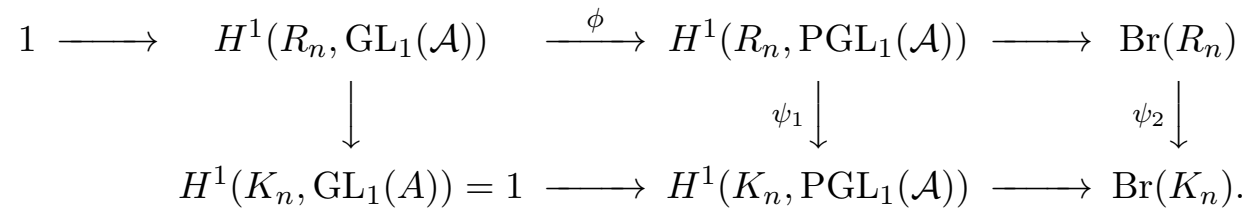

Every class in $H^{1}\left(R_{n}, \mathrm{GL}_{1}(\mathcal{A})\right.$ is rationally trivial, hence by Fedorov-Panin's result [FP, $\mathrm{PSV}]$ it is locally trivial in the Zariski topology. In other words

$$
H^{1}\left(R_{n}, \mathrm{GL}_{1}(\mathcal{A})\right)=H_{Z a r}^{1}\left(R_{n}, \mathrm{GL}_{1}(\mathcal{A})\right) .
$$

Clearly

$$
\phi\left(H_{Z a r}^{1}\left(R_{n}, \mathrm{GL}_{1}(\mathcal{A})\right)\right) \subseteq H_{Z a r}^{1}\left(R_{n}, \mathrm{PGL}_{1}(\mathcal{A})\right) .
$$

Conversely, let $\gamma \in H_{Z a r}^{1}\left(R_{n}, \operatorname{PGL}_{1}(\mathcal{A})\right)$. Since $\psi_{1}(\gamma)=1$ and since $\psi_{2}$ is injective, we obtain $\gamma \in \operatorname{Im}(\phi)$.

Finally, it remains to note that the above diagram shows that $\phi$ has trivial kernel and this is true for all Azumaya algebras over $R_{n}$. Then the standard twisting argument enables us to conclude that $\phi$ is injective.

Thus, the disjoint union decomposition of the set of isomorphism classes of torsors under $\mathrm{PGL}_{d}$ becomes

$$
\bigsqcup_{[\mathcal{A}] \in H_{\text {toral }}^{1}\left(R_{n}, \mathrm{PGL}_{d}\right)} H_{Z a r}^{1}\left(R_{n}, \mathrm{GL}_{1}(\mathcal{A})\right) \stackrel{\stackrel{\Theta}{\sim}}{\longrightarrow} H^{1}\left(R_{n}, \mathrm{PGL}_{d}\right)
$$


In general we can't say much about the subset $H_{Z a r}^{1}\left(R_{n}, \mathrm{GL}_{1}(\mathcal{A})\right)$. Recall only that it classifies right invertible $\mathcal{A}$-modules (so that the above decomposition is coherent with GP2, prop. 4.8]). More precisely, if $\mathcal{A}$ is a toral Azumaya $R_{n}$-algebra and $\mathcal{L}$ is a right invertible $\mathcal{A}$-module, then the class $[\mathcal{L}]$ corresponds to the class of the Azumaya algebra $\operatorname{End}_{\mathcal{A}}(\mathcal{L})$ under the map $\Theta$.

However, when the base field $k$ is algebraically closed, toral Azumaya algebras over $R_{n}$ are easy to classify explicitly, and also much more information about Zariski trivial torsors is available due to Artamonov's freeness statements $\mathrm{A}$.

More precisely, let $k$ be an algebraically closed field. Choose a coherent system of primitive roots of unity $\left(\zeta_{n}\right)_{n \geq 1}$ in $k$. Given integers $r, s$ satisfying $1 \leq r \leq s$, we let $A(x, y)_{r}^{s}$ denote the Azumaya algebra of degree $s$ over the Laurent polynomial ring $k\left[x^{ \pm 1}, y^{ \pm 1}\right]$ defined by a presentation

$$
X^{s}=x, Y^{s}=y, Y X=\zeta_{s}^{r} X Y .
$$

5.11. Lemma. Let $s_{1}, \ldots, s_{m}, r_{1}, \ldots r_{m}$ be positive integers such that $\left(s_{i}, r_{i}\right)=$ 1 for all $i=1, \ldots, m$. Then the Azumaya algebra

$$
\mathcal{A}=A\left(t_{1}, t_{2}\right)_{r_{1}}^{s_{1}} \otimes A\left(t_{3}, t_{4}\right)_{r_{2}}^{s_{2}} \otimes \cdots A\left(t_{2 m-1}, t_{2 m}\right)_{r_{m}}^{s_{m}}
$$

is a division algebra.

Proof. Indeed, using the residue method it is easy to see that it is a division algebra even over the field $F_{2 m}=k\left(\left(t_{1}\right)\right) \ldots\left(\left(t_{2 m}\right)\right)$.

We next recall that the group $\mathrm{GL}_{d}(\mathbb{Z})$ acts in a natural way on the ring $R_{n}$, hence it acts on $H^{1}\left(R_{n}, \mathrm{PGL}_{d}\right)$ (for details see [GP3, 8.4]).

5.12. Theorem. Assume that $k$ is algebraically closed.

(1) $H_{\text {toral }}^{1}\left(R_{n}, \mathrm{PGL}_{d}\right)$ consists of $\mathrm{GL}_{d}(\mathbb{Z})$-orbits of classes of $R_{n}$-algebras of the following shape:

$$
\mathcal{A}=M_{s_{0}}\left(R_{n}\right) \otimes_{R_{n}} A\left(t_{1}, t_{2}\right)_{r_{1}}^{s_{1}} \otimes A\left(t_{3}, t_{4}\right)_{r_{2}}^{s_{2}} \otimes \cdots A\left(t_{2 m-1}, t_{2 m}\right)_{r_{m}}^{s_{m}}
$$

where the integers $m, s_{0}, s_{1}, r_{1}, \ldots, s_{m}, r_{m}$ satisfy the following conditions:

(i) $0 \leq 2 m \leq n ; 1 \leq r_{i} \leq s_{i}$ and $\left(r_{i}, s_{i}\right)=1$ for all $i=1, \ldots, m$;

(ii) $s_{0} \geq 1$ and $s_{0} s_{1} \ldots s_{m}=d$.

(2) $\mathcal{A} \otimes_{R_{n}} F_{n}$ is division if and only if $s_{0}=1$.

(3) Let $\mathcal{A}$ be an Azumaya algebra as in (1). If $s_{0} \geq 2$ we have

$$
H_{Z a r}^{1}\left(R_{n}, \mathrm{GL}_{1}(\mathcal{A})\right)=1 \text {. }
$$

Proof. (1) If $\mathcal{A} \otimes_{R_{n}} F_{n}$ is division, this is [GP3, th. 4.7]. Assume now that $\mathcal{A} \otimes_{R_{n}} F_{n}$ is not division. By Wedderburn's theorem there exists an integer $s \geq 1$ and a central division $F_{n}$-algebra $A^{\prime}$ such that $\mathcal{A} \otimes_{R_{n}} F_{n} \cong M_{s}\left(A^{\prime}\right)$. The acyclicity theorem provides a toral $R_{n}$-Azumaya algebra $\mathcal{A}^{\prime}$ such that $\mathcal{A}^{\prime} \otimes_{R_{n}} F_{n} \cong A^{\prime}$. Since $M_{s}\left(\mathcal{A}^{\prime}\right)$ and $\mathcal{A}$ are isomorphic over $F_{n}$, the acyclicity theorem again shows that $\mathcal{A} \cong M_{s}\left(\mathcal{A}^{\prime}\right)$. It remains to note that $\mathcal{A}^{\prime}$ being a division algebra over $R_{n}$ is of the required form by the first case. 
(2) The assertion follows from Lemma 5.11.

(3) Write $\mathcal{A}=M_{s}\left(\mathcal{A}^{\prime}\right)$ with $\mathcal{A}^{\prime}$ is division and $s \geq 2$. Morita equivalence provides a one-to-one correspondence between invertible $\mathcal{A}$-modules and finitely generated projective $\mathcal{A}^{\prime}$-modules of relative rank $s$. Artamonov's result [A] states that those $\mathcal{A}^{\prime}$-modules are free $[\mathrm{A}$, cor. 3] since $s \geq 2$, so that invertible $\mathcal{A}$-modules are free as well. This implies $H_{Z a r}^{1}\left(R_{n}, \mathrm{GL}_{1}(\mathcal{A})\right)=1$.

5.13. Remarks. (a) The third statement shows that for each invertible $\mathcal{A}-$ module $P$ the module $P \oplus \mathcal{A}$ is free.

(b) The third statement refines Steinmetz's results in the 2-dimension case $(n=2)[$ St, th. 4.8] where the case $s \geq 3$ was considered only. Note that St provides some other cases for classical groups when all Zariski locally trivial torsors are trivial.

5.14. Applications to $R_{n}$-Lie algebras. We next consider the special case $\mathbf{G}=\operatorname{Aut}(\mathfrak{g})$ where $\mathfrak{g}$ is a split simple Lie algebra over $k$ of finite dimension. For such group the set $H_{e ̂ t}^{1}\left(R_{n}, \mathbf{G}\right)$ classifies $R_{n}$-forms of the Lie algebra $\mathfrak{g} \otimes_{k} R_{n}$ and $H_{\text {toral }}^{1}\left(R_{n}, \mathbf{G}\right)$ classifies loop objects, i.e. those which arise from loop cocycles. More precisely, by [GP3, §6] we have

$$
\operatorname{Im}\left(H^{1}\left(\pi_{1}\left(R_{n}, 1\right), \mathbf{G}\left(k_{s}\right)\right) \rightarrow H^{1}\left(R_{n}, \mathbf{G}\right)\right)=H_{\text {toral }}^{1}\left(R_{n}, \mathbf{G}\right) .
$$

Theorems 1.2 and 5.2 have the following consequences.

5.15. Corollary. (1) Let $\widetilde{\mathcal{L}}$ be a $K_{n}$-form of the Lie algebra $\mathfrak{g} \otimes_{k} K_{n}$. If $\widetilde{\mathcal{L}}$ is unramified, i.e. extends everywhere in codimension one, then $\widetilde{\mathcal{L}}$ is isomorphic to the generic fiber of a unique multiloop Lie algebra $\mathcal{L}$. (Of course $\mathcal{L}$, being a multiloop algebra, is a twisted from of the $R_{n}$-Lie algebra $\left.\mathfrak{g} \otimes_{k} R_{n}\right)$.

(2) Let $\mathcal{L}$ be any $R_{n}$-form of $\mathfrak{g} \otimes_{R} R_{n}$. Then there exists a (unique up to $R_{n^{-}}$ isomorphism) multiloop Lie algebra $\mathcal{L}_{\text {loop }}$ over $R_{n}$ such that $\mathcal{L}$ is a Zariski $R_{n}$-form of $\mathcal{L}_{\text {loop }}$.

\section{REFERENCES}

[A] V. A. Artamonov, Projective modules over crossed products, J. Algebra 173 (1995), 696-714.

[Bbk] N. Bourbaki, Algèbre commutative, Ch. 1 à 4, Springer.

[CF] B. Calmès, J. Fasel, Groupes classiques, to appear in Autour des schémas en groupes, vol II, Panoramas et Synthèses (2015).

[CGP1] V. Chernousov, P. Gille and A. Pianzola, Torsors over the punctured affine line, American Journal of Mathematics 134 (2012), 1541-1583.

[CGP2] V. Chernousov, P. Gille and A. Pianzola, Conjugacy theorems for loop reductive group schemes and Lie algebras, Bulletin of Mathematical Sciences 4 (2014), 281-324.

[CT] J.-L. Colliot-Thélène, Birational invariants, purity and the Gersten conjecture, Ktheory and algebraic geometry: connections with quadratic forms and division algebras (Santa Barbara, CA, 1992), 1-64, Proc. Sympos. Pure Math. 58.1 (1995), AMS. 
[CTO] J.-L. Colliot-Thélène, M. Ojanguren, Espaces principaux homogènes localement triviaux, I.H.É.S. Publ. Math. 75 (1992), 97-122.

[CTS] J.-L. Colliot-Thélène, J.-J. Sansuc, Fibrés quadratiques et composantes connexes réelles, Math. Annalen 244 (1979), 105-134.

[DG] M. Demazure et P. Gabriel, Groupes algébriques, Masson (1970).

[EKM] R. Elman, N. Karpenko, A. Merkurjev, The algebraic and geometric theory of quadratic forms, American Mathematical Society Colloquium Publications, 56. American Mathematical Society, Providence, RI, 2008.

[EGAI] A. Grothendieck (avec la collaboration de J. Dieudonné), Eléments de Géométrie Algébrique I: Le langage des schémas, Publications mathématiques de l'I.H.É.S. no 4 (1960).

[EGAIV] A. Grothendieck (avec la collaboration de J. Dieudonné), Eléments de Géométrie Algébrique IV: Étude locale des schémas et des morphismes de schémas, Publications mathématiques de l'I.H.É.S. no 20, 24, 28 and 32 (1964-1967).

[FP] R. Fedorov, I. Panin, A proof of Grothendieck-Serre conjecture on principal bundles over regular local rings containing infinite fields, to appear in Publications Mathématiques de l'I.H.É.S.

[GP1] P. Gille and A. Pianzola, Galois cohomology and forms of algebras over Laurent polynomial rings, Mathematische Annalen 338 (2007), 497-543.

[GP2] P. Gille and A. Pianzola, Isotriviality and étale cohomology of Laurent polynomial rings, J. Pure Appl. Algebra 212 (2008), 780-800.

[GP3] P. Gille and A. Pianzola, Torsors, reductive group schemes and extended affine Lie algebras, Memoirs of AMS 1063 (2013).

[Gi] J. Giraud, Cohomologie non abélienne, Die Grundlehren der mathematischen Wissenschaften 179 (1971), Springer-Verlag.

[Gr] A. Grothendieck, Le groupe de Brauer. II, Théorie cohomologique (1968), Dix Exposés sur la Cohomologie des Schémas, 67-87, North-Holland.

[KLP] V. Kac, M. Lau and A. Pianzola. Differential conformal superalgebras and their forms, Advances in Mathematics 222 (2009), 809-861.

[M] J.S. Milne, Etale cohomology, Princeton University Press (1980).

[N] Y. A. Nisnevich, Espaces homogènes principaux rationnellement triviaux et arithmétique des schémas en groupes réductifs sur les anneaux de Dedekind, C. R. Acad. Sci. Paris Sér. I Math. 299 (1984), 5-8.

[PSV] I. Panin, A. Stavrova and N. Vavilov, On Grothendieck-Serres conjecture concerning principal G-bundles over reductive group schemes: I, Compositio Math. 151 (2015), 535-567.

[PZ] G. Pappas, X. Zhu, Local models of Shimura varieties and a conjecture of Kottwitz, Inventiones Mathematicae 194 (2013), 147-254.

[P] R. Parimala, Quadratic spaces over Laurent extensions of Dedekind domains, Trans. Amer. Math. Soc. 277 (1983), 569-578.

[R] M.S. Raghunathan, Principal bundles on affine space and bundles on the projective line, Math. Ann. 285 (1989), 309-332.

[Se] J.-P. Serre, Espaces fibrés algébriques, Séminaire Claude Chevalley 3 (1958), Exposé no. $1,37 \mathrm{p}$.

[SGA1] Séminaire de Géométrie algébrique de l'I.H.É.S., Revêtements étales et groupe fondamental, dirigé par A. Grothendieck, Lecture Notes in Math. 224 (1971), Springer.

[SGA3] Séminaire de Géométrie algébrique de l'I.H.É.S., 1963-1964, schémas en groupes, dirigé par M. Demazure et A. Grothendieck, Lecture Notes in Math. 151-153 (1970), Springer.

[St] A. Steinmetz-Zikesch, Algèbres de Lie de dimension infinie et théorie de la descente, Mémoire de la SMF 129 (2012). 
Department of Mathematics, University of Alberta, Edmonton, Alberta T6G 2G1, CANADA

E-mail address: chernous@math.ualberta.ca

UMR 5208 Institut Camille Jordan - Université Claude Bernard Lyon 143 Boulevard du 11 novembre 191869622 Villeurbanne CEdex - France Institute of Mathematics Simion Stoilow of the Romanian Academy, Calea Grivitei 21, RO-010702 Bucharest, Romania.

E-mail address: gille@math.univ-lyon1.fr

Department of Mathematics, University of Alberta, Edmonton, Alberta T6G 2G1, CANADA.

Centro de Altos Estudios en Ciencia Exactas, Avenida de Mayo 866, (1084) Buenos Aires, Argentina.

E-mail address: a.pianzola@gmail.com 